\title{
Internal structure of localized quantized vortex tangles
}

\section{Tomo Nakagawa, Sosuke Inui, Makoto Tsubota}

\begin{tabular}{|c|l|}
\hline Citation & PHYSICAL REVIEW B. 104(9); 094510. \\
\hline Issue Date & 2021-09-09 \\
\hline Type & Journal Article \\
\hline Textversion & Publisher \\
\hline Rights & $\begin{array}{l}\text { O2021 American Physical Society. For personal use only. No other uses without } \\
\text { permission. }\end{array}$ \\
\hline DOI & 10.1103/PhysRevB.104.094510 \\
\hline
\end{tabular}

Self-Archiving by Author(s)

Placed on: Osaka City University Repository 


\title{
Internal structure of localized quantized vortex tangles
}

\author{
Tomo Nakagawa $\odot,{ }^{1}$ Sosuke Inui $\odot,{ }^{1}$ and Makoto Tsubota ${ }^{1,2}$ \\ ${ }^{1}$ Department of Physics, Osaka City University, 3-3-138 Sugimoto, 558-8585 Osaka, Japan \\ ${ }^{2}$ Department of Physics, Nambu Yoichiro Institute of Theoretical and Experimental Physics, and OCU Advanced Research Institute for \\ Natural Science and Technology, Osaka City University, 3-3-138 Sugimoto, Osaka 558-8585, Japan
}

(Received 19 July 2021; revised 20 August 2021; accepted 27 August 2021; published 9 September 2021)

\begin{abstract}
In this study, we numerically investigate the internal structure of localized quantum turbulence in superfluid ${ }^{4} \mathrm{He}$ at zero temperature with the expectation of self-similarity in the real space. In our previous study, we collected the statistics of vortex rings emitted from a localized vortex tangle. As a result, the power law between the minimum size of detectable vortex rings and the emission frequency is obtained, which suggests that the vortex tangle has self-similarity in the real space [Nakagawa et al., Phys. Rev. B 101, 184515 (2020)]. In this work, we study the fractal dimension and vortex length distribution of localized vortex tangles, which can show their self-similar structure. We generate statistically steady and localized vortex tangles by injecting vortex rings with a fixed size. We used two types of injection methods that produce anisotropic or isotropic tangles. The injected vortex rings develop into a localized vortex tangle consisting of vortex rings of various sizes through reconnections (fusions and splitting of vortices). The fractal dimension is an increasing function of the vortex line density and becomes saturated to a value of approximately 1.8 , as the density increases sufficiently. The behavior of the fractal dimension was independent of the anisotropy of the vortex tangles. The vortex length distribution indicates the number of vortex rings of each size that are distributed in a tangle. The distribution of the anisotropic vortex tangle shows the power law in the range above the injected vortex size, although the distribution of the isotropic vortex does not.
\end{abstract}

DOI: 10.1103/PhysRevB.104.094510

\section{INTRODUCTION}

Turbulence is one of the most important problems in physics, and many researchers have studied it for many years $[1,2]$. It is a typical phenomenon that is nonequilibrium and nonlinear, which prevents us from understanding it well. Quantum turbulence is often considered as a clue to resolve the nonequilibrium and nonlinear phenomena of turbulence. In classical turbulence or conventional fluid turbulence, it is difficult to identify vortices, which are important elements of turbulence, because they are unstable and not well defined. However, in quantum turbulence, namely, turbulence in quantum condensed fluids, vortices are well-defined topological defects and have quantized circulation. Therefore, studies on quantum turbulence provide a shortcut to understanding turbulence [3-6].

Superfluid ${ }^{4} \mathrm{He}$ is a typical quantum fluid. Liquid ${ }^{4} \mathrm{He}$ transitions to the superfluid phase below the temperature $T_{\lambda}=$ $2.17 \mathrm{~K}$. The physics of superfluid ${ }^{4} \mathrm{He}$ is described by the two-fluid model: The system consists of a superfluid component and a normal fluid component $[7,8]$. The superfluid component has no viscosity and entropy, whereas the normal fluid component has both. The ratio of the densities depended on the temperature. When the temperature was below $1 \mathrm{~K}$, the normal fluid component almost disappeared. In the superfluid component, the circulation is quantized to $\kappa=h / m$, where $h$ and $m$ are the Planck constant and the mass of a ${ }^{4} \mathrm{He}$ atom, respectively [9]. A typical quantum turbulence consists of a tangle of quantized vortices.

In turbulence, the velocity field is disturbed both spatially and temporally. Therefore, researchers have tried to describe turbulence not by the velocity field but by universal and reproducible statistical laws. In classical turbulence, Kolmogorov's law is well known as the universal law showing self-similarity in the wave-number space. This law implies that the energy spectrum of isotropic and homogeneous turbulence is proportional to the wave number to the power of $-5 / 3$, and its energy is transferred from low wave numbers to high wave numbers. Kolmogorov's law has also been studied and confirmed in quantum turbulence [10-15]. However, the self-similarity in the real space is not well understood because the vortices are not well defined in classical turbulence, although several self-similar models have been studied. In quantum turbulence, all vortices are well defined, which enables us to consider the self-similarity in the real space clearly.

The quantum turbulence of superfluid ${ }^{4} \mathrm{He}$ has been studied for more than half a century. Most efforts of researches have been devoted to thermal counter flow [3,4]. However, in recent years, localized turbulence, such as that generated by oscillating objects, has received considerable attention [16-27]. A typical example of an oscillating object is a vibrating wire. Yano et al. conducted wire experiments and found some statistical laws for localized vortex tangles $[18,23,27]$. They generated the vortex tangle by the vibrating wires and detected 
vortex rings emitted from the tangle. They could manage the minimum size of detectable vortex rings by varying the temperature and obtain the statistics on the size of vortex rings emitted from the tangle. They found a power law between the minimum size of detectable vortex rings and their detection frequencies. It may show self-similarity in the real space because the emitted vortex rings provide information of the structure of the tangle where they belong. Yano et al. discussed that the power law is related to a fractal dimension of the vortex tangle [27].

Our goal is to understand self-similarity in the real space of localized vortex tangles. In our previous study [28], we investigated vortex rings emitted from vortex tangles and obtained results qualitatively similar to the experiments in Ref. [27], such as the self-similarity of the vortex emission.

In this study, we have developed the previous study and investigated the internal structure of vortex tangles with the expectation of self-similarity. To accomplish this goal, we have studied the fractal dimension [29,30] and the vortex length distribution [31,32], which may reveal the selfsimilarity of vortex tangles directly: The fractal dimension is a noninteger dimension that characterizes a self-similar structure. This dimension was calculated by Kivotides et al . for vortex tangles in periodic boundary conditions [30]. The vortex length distribution is a probability density function of the vortex rings in a vortex tangle depending on their size. The distribution is expected to have the information of the internal structure of the tangle from which vortex rings are emitted. In Secs. III and IV, we describe them in detail.

We also investigated the universality independent of the anisotropy of the tangles. We made the tangles using two methods numerically. One is the same method as in our previous study, which makes anisotropic localized vortex tangles [28]. The other is to create isotropic localized vortex tangles. The two methods enable us to investigate the universality independent of the anisotropy of the tangle.

This paper comprises five sections. In Sec. II, we introduce the vortex filament model and the system that we consider. Section III presents the results of calculating the fractal dimensions. In Sec. IV, we discuss the vortex length distribution. Finally, the conclusions are presented in Sec. V.

\section{THE MODEL AND SYSTEM}

\section{A. Vortex filament model}

The circulation of vortices in superfluid ${ }^{4} \mathrm{He}$ is quantized, and the cores have very thin structures of the order of $1 \AA$. For these reasons, the thin structures of the vortex cores can be neglected, and a vortex filament model is available [33]. In this study, we performed a simulation of the dynamics of the vortex filament model at $T=0 \mathrm{~K}$. The superfluid velocity field obtained by the filaments obeys the Biot-Savart law. The equation of motion of the vortex filaments is:

$$
\frac{d \boldsymbol{s}}{d t}=\frac{\kappa}{4 \pi} \int_{\mathcal{L}} \frac{\boldsymbol{s}^{\prime}(\xi, t) \times[\boldsymbol{r}-\boldsymbol{s}(\xi, t)]}{|\boldsymbol{r}-\boldsymbol{s}(\xi, t)|^{3}} d \xi,
$$

where $s(\xi, t)$ is the position of the filaments represented by the parameter $\xi$, and $s^{\prime}$ is $\partial s / \partial \xi$. The integration is performed over all vortex filaments $\mathcal{L}$.
To calculate this equation numerically, we discretized the vortex filaments into points and the separation $\Delta \xi$ is set to be within the range of $\Delta \xi_{\min }=0.5 \mu \mathrm{m}$ and $\Delta \xi_{\max }=1.2 \mu \mathrm{m}$. The time resolution is $\Delta t=5 \times 10^{-6} \mathrm{~s}$ and the temporal integration of Eq. (1) is solved by the fourth-order Runge-Kutta method. As this model cannot describe the reconnection of vortices, we make them reconnect algorithmically when their distance becomes less than $\Delta \xi_{\text {min }}$. We deleted small vortex rings with sizes smaller than $5 \Delta \xi_{\text {min }}$.

\section{B. The systems}

We generate localized vortex tangles by two different methods, "parallel injection" and "spherical injection." The advantage of these methods is that they can locally generate a steady vortex tangle. The steady state of the vortex tangle indicates the equilibrium between the excitation and the dissipation in a finite volume because we are interested in a localized vortex tangle. The method of determining the volume occupied by the tangle depends on the injection methods and will be introduced later. The excitation originates from vortex injections. There are two types of dissipation: The vortex ring escaping from the finite volume and the deletion of the small vortex rings. The ratios of these two types of dissipation were roughly the same.

\section{Parallel injection}

The parallel injection method was used in our previous study [28]. In this model, two parallel square vortex sources are prepared, and vortex rings are injected from a random position of the two sources simultaneously with a certain frequency $f$ to collide them and generate a localized tangle. The parameters of this model are the injection frequency $f$ and diameter $2 R_{0}$ of the injected rings. The vortex tangles made by this method are anisotropic, as shown in Figs. 1(a) and 1(b). In this system, the vortex line length in a finite volume covering the tangle becomes statically steady after a certain time, as shown in Fig. 2(a). The finite volume we applied is a cylindrical volume with a height of $160 \mu \mathrm{m}$ and a radius of $250 \mu \mathrm{m}$. As we are interested only in the localized tangle, we deleted the vortex rings that escape far from the tangle.

\section{Spherical injection}

In the spherical injection method, we inject vortex rings from the virtual spherical source of radius $180 \mu \mathrm{m}$. An example of the tangle is shown in Figs. 1(c) and 1(d). The parameters are similar to those of the former method. With a fixed frequency $f$, two vortex rings with size $2 R_{0}$ are injected from random positions on the source. The tangles in a finite volume become statistically steady after a certain time, as shown in Fig. 2(b). In this method, the applied finite volume is a spherical volume with a radius of $180 \mu \mathrm{m}$. We also deleted vortex rings that are far from the tangle.

The vortex line density distributions of the tangles are shown in Fig. 3. The tangle generated by parallel injection spreads out in the $y$ and $z$ directions and is relatively flat along the $x$ axis. However, that by spherical injection is isotropic. The purpose of using the two methods is to determine the universality independent of the anisotropy. 


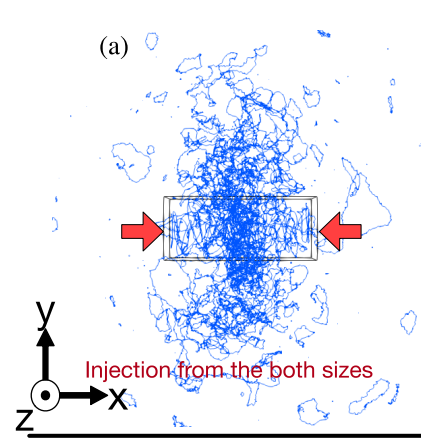

(c)

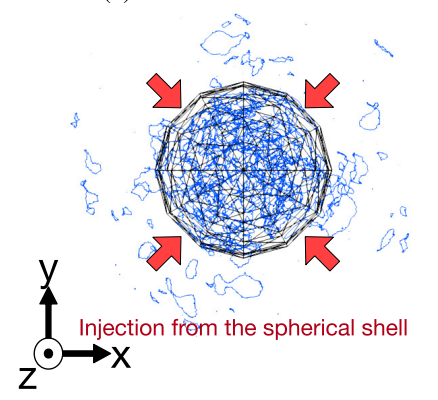

(d)

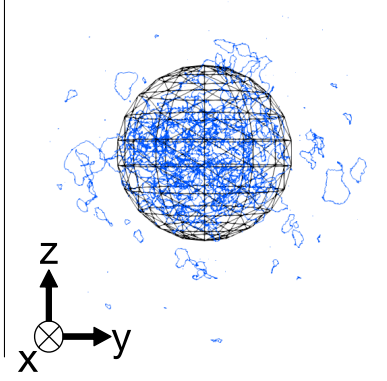

FIG. 1. The vortex tangles generated by the vortex injection at time $0.2 \mathrm{~s}$. Panels (a) and (b) are the vortex tangle generated by parallel injection in the condition $2 R_{0}=60 \mu \mathrm{m}$ and $f=1000 \mathrm{~Hz}$. Moreover, (c) and (d) are the tangle generated by spherical injection in the condition $2 R_{0}=60 \mu \mathrm{m}$ and $f=1000 \mathrm{~Hz}$.
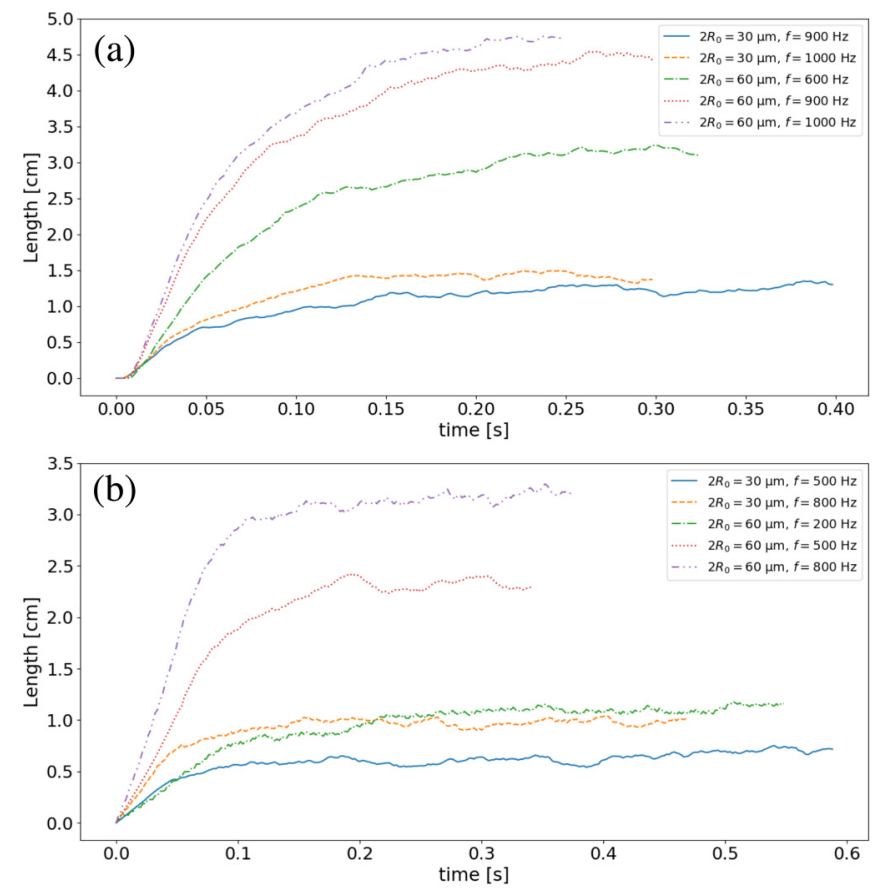

FIG. 2. Time development of the vortex line length. Panel (a) is the tangle generated by parallel injection and (b) is generated by "spherical injection." The length is calculated in the finite volume. In the case of "parallel injection," the volume is the cylindrical volume with height $160 \mu \mathrm{m}$ and radius $250 \mu \mathrm{m}$. The volume in spherical injection is the spherical volume with radius $180 \mu \mathrm{m}$. Both volumes cover the vortex tangles
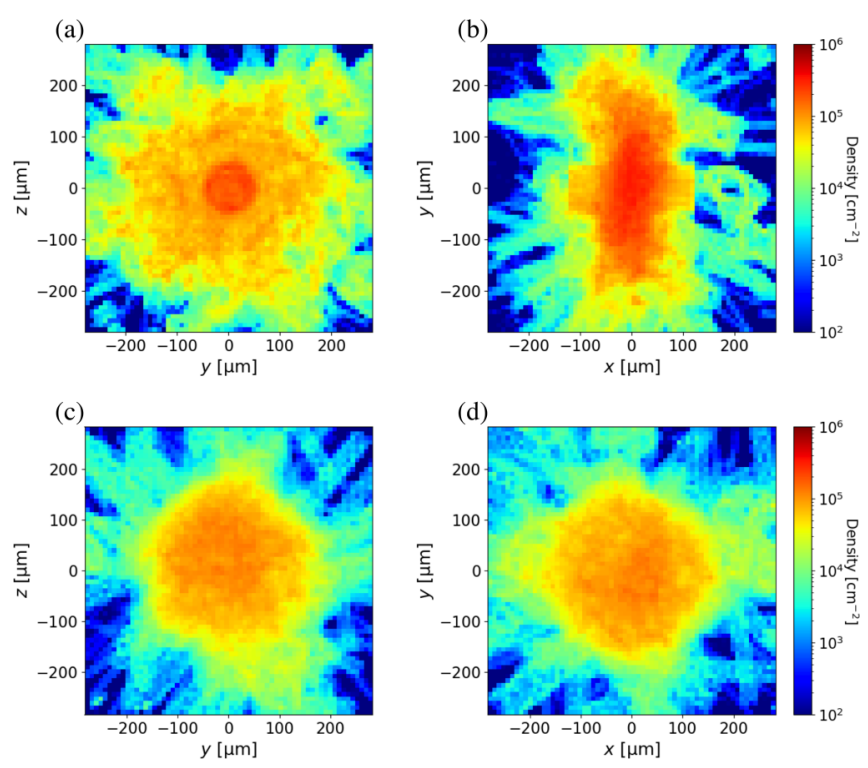

FIG. 3. Time-averaged vortex line density distribution of the vortex tangles. Panels (a) and (b) are the distribution of "parallel injection," while (c) and (d) are the distribution of "spherical injection." The vortex line density is calculated in $280 \mu \mathrm{m} \times \Delta y \times \Delta z$ in (a) and (c). Panels (b) and (d) are the vortex line density in $\Delta x \times \Delta y \times 280 \mu \mathrm{m}$. Here, $\Delta x=\Delta y=\Delta z=280 / 64 \mu \mathrm{m}$.

\section{FRACTAL DIMENSION}

A fractal dimension is a noninteger dimension that represents how the fine structure of a pattern changes with the measured scale defined by

$$
N \propto k^{D_{f}},
$$

where $k$ is a length scale and $N$ is the number of structures with scale $k$. We obtain the fractal dimension of our vortex tangles using the box-counting method $[29,30]$. In this method, we divide a virtual box with the size of the system into a scale of length $\delta$ and count the number $N(\delta)$ of the boxes covering the tangle. Therefore, the fractal structure should show the following relation:

$$
D_{f}=-\frac{\ln \left(N(\delta) / N_{\min }\right)}{\ln \left(\delta / \delta_{\min }\right)},
$$

where $\delta_{\min }$ is the minimum scale of length of the fitting range, and $N_{\min }$ is $N\left(\delta_{\min }\right)$. By plotting $\delta$ and $N(\delta)$ in a log-log scale and calculating its slope, we can determine the fractal dimension of the pattern. In this calculation, the maximum size of the box is set to $800 \mu \mathrm{m}$ per side.

Regarding the fractal dimension of quantum turbulence, there is a previous study [30]. Kivotides et al. prescribed a normal fluid turbulent flow and generated vortex tangles numerically through mutual friction under periodic conditions. Then, they obtained the fractal dimension of the vortex tangles with a vortex line density of $0.05-0.20 \times 10^{5} \mathrm{~cm}^{-2}$ by the box-counting method and discovered that the dimension $D_{f}$ is an increasing function of the vortex line density $L$ defined by

$$
L=\frac{1}{V} \int_{\mathcal{L}^{\prime}} d \xi
$$




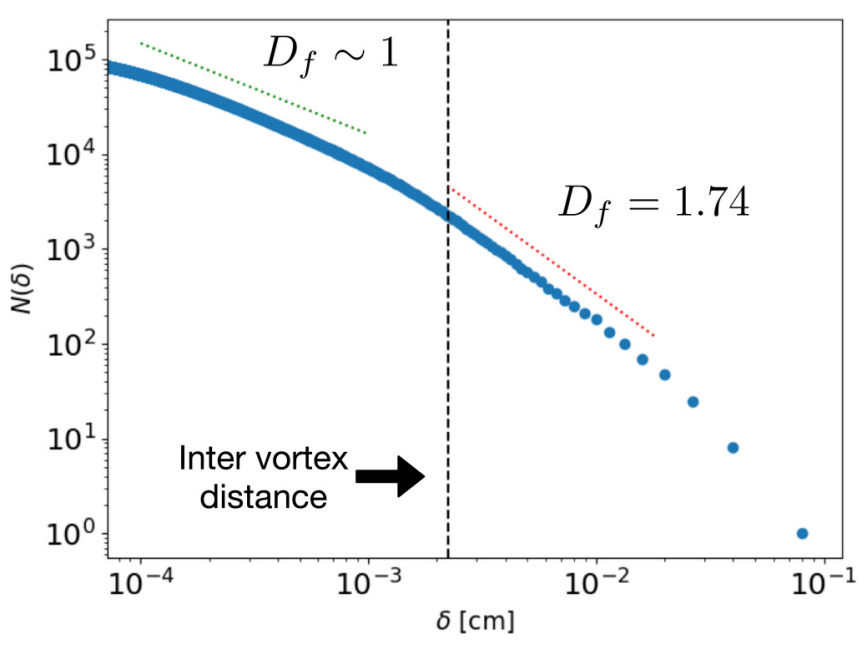

FIG. 4. The plotting of $\delta$ and $N(\delta)$ applying the box-counting method to the tangle by "parallel method" in the condition $2 R_{0}=$ $60 \mu \mathrm{m}$ and $f=1000 \mathrm{~Hz}\left(\delta_{\min }=2.22 \times 10^{-3} \mathrm{~cm}\right.$ and $\left.N_{\min }=2526\right)$. The green line shows the slope in the small scale (from the inter vortex distance to the space resolution). The red line shows the slope in the middle scale (up to the intervortex distance).

Here, the integration is performed over the path that corresponds to the filaments $\mathcal{L}^{\prime}$ within a volume $V$. In the study, they set the system size to be $V$. Their values of $D_{f}$ were roughly 1.4-1.7.

In this study, we calculate the fractal dimension of localized vortex tangles using the box-counting method. Figure 4 shows the typical result in a statistical steady state of "parallel injection." In small scales, it shows $D_{f} \approx 1$, which refers to the dimension of each vortex filament as a one-dimensional object. When the scale exceeds a certain critical value, $D_{f}$ changes to another value larger than unity, which is the fractal dimension of the tangle. This behavior is confirmed for all vortex tangles produced under different conditions. We define the value at the large scale the fractal dimension $D_{f}$ of the vortex tangle.

The transition from a small scale to a large scale is determined by the mean intervortex distance. The distance is the square root of the inverse of the vortex line density calculated by Eq. (4). In this study, we set the volume $V$ in Eq. (4) to be the finite volume depending on each injection method, as mentioned in Sec. II(B).

Figure 5 shows the time development of $D_{f}$ for 'parallel injection. In earlier times, they fluctuate because the systems have a small number of vortices that it is difficult to calculate the fractal dimension of vortex tangles properly.

However, at a later time, $D_{f}$ becomes stationary and asymptotically approaches a certain value in each condition, which indicates that the tangles become statistically steady. Figure 6 shows the time-averaged $D_{f}$ after $0.2 \mathrm{~s}$ as a function of vortex line density. In the low-density range, $D_{f}$ increases with vortex line density. This behavior is consistent with the results of Kivotides et al. [30]. In the higher density range, the fractal dimensions converge to $D_{f} \sim 1.8$, regardless of the injection method. Although the tangles have completely different shapes depending on their injection method, the tangles have similar fractal dimensions. This is nontrivial.

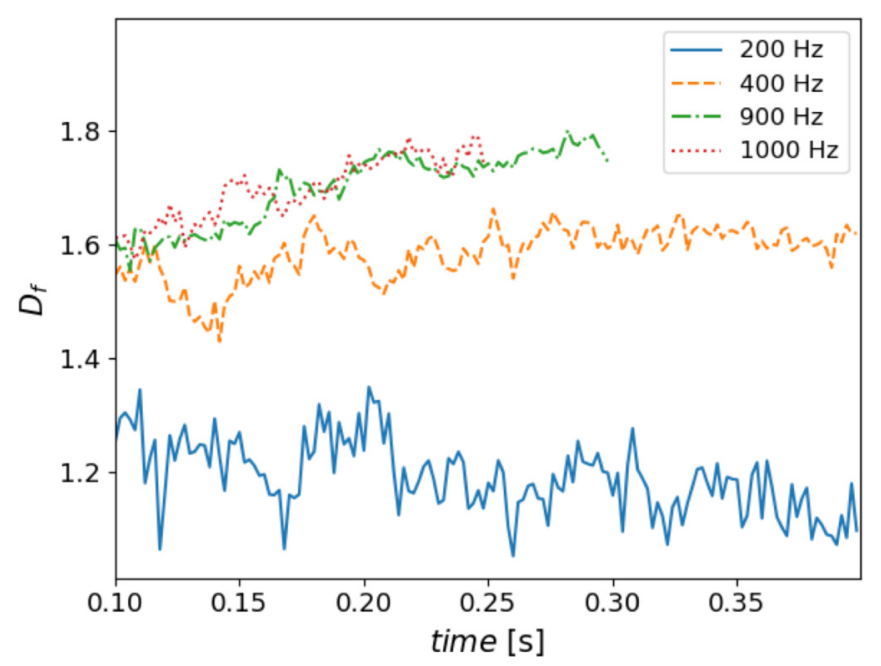

FIG. 5. The time development of the fractal dimension of the vortex tangles generated by parallel injection in the conditions $2 R_{0}=$ $60 \mu \mathrm{m}$ and some injection frequencies $f$.

The developed vortex tangles construct intermittent structures on their own owing to the interaction between vortices, and the tangles show similar fractal dimensions $D_{f} \sim 1.8$. The values of the fractal dimension roughly agree with the results of Kivotides et al. [30]. However, it is difficult to make a quantitative comparison because the systems are different. The three main differences are summarized in Table I. First, the crucial difference is temperature. Kivotides et al. studied the vortex dynamics at finite temperatures, where the mutual friction affected the motion of the vortices [30]. Second, they prescribed turbulent normal flow [34] and excited the vortices through the mutual friction; we generated vortex tangles by injecting vortices. Third, the boundary condition of Ref. [30] was periodic, but in this study it is not applied.

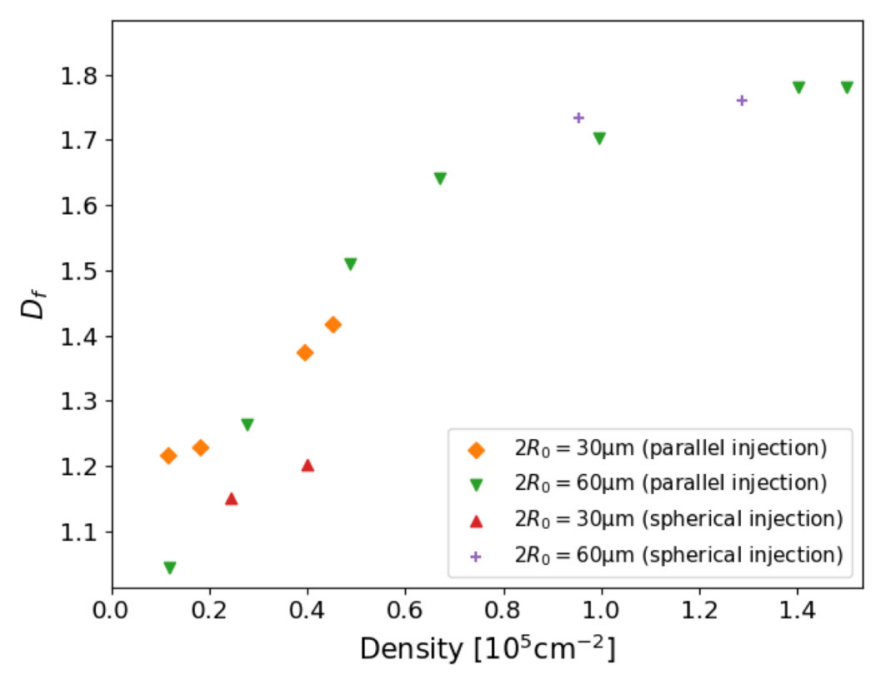

FIG. 6. The relation between the vortex line density and the fractal dimension. The dimension becomes saturated as the vortex line density increases. 
TABLE I. Comparison of the systems of this study and the previous study [30].

\begin{tabular}{lcc}
\hline \hline & This study & Kivotides et al. [30] \\
\hline Temperature & $0 \mathrm{~K}$ & $1.3 \mathrm{~K}, 1.9 \mathrm{~K}$ \\
Normal fluid velocity & Not relevant & Turbulent flow [34] \\
Boundary condition & N/A & Periodic \\
\hline \hline
\end{tabular}

\section{VORTEX LENGTH DISTRIBUTION}

In this calculation, all vortices are closed loops because the system is free from solid boundaries. The vortex length distribution is the probability density function of the existence of vortex rings in the tangle depending on their sizes. The distribution has been studied previously [31,32], especially with the expectation that it shows a self-similar structure. Fujiyama and Tsubota found that the distribution obeys a self-similar distribution and corresponds to the fluctuation of the vortex line density [32]. In this study, we collect data on the lengths of rings and investigated the distribution and dependence on the anisotropy of the tangles.

The time developments of the distribution for parallel injection and spherical injection are shown in Figs. 7(a) and 7 (b). The lengths are normalized by $2 \pi R_{0}$. In earlier times, the distribution concentrates in unity, referring to the initial

(a)

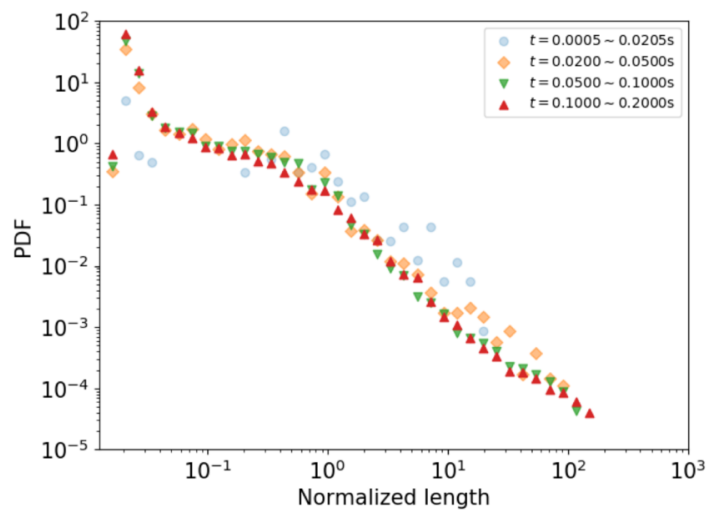

(c)

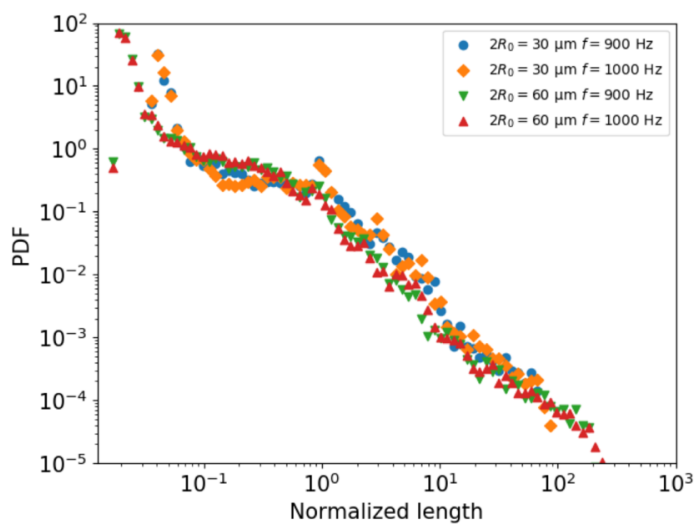

injection vortices. The distributions develop into both smaller and larger than the injected length, which shows that the injected vortices split into smaller ones and merge among them to become larger ones. After a long time, it converges to a statistically steady distribution.

It seems that the distributions are saturated just before their vortex line lengths reach a roughly constant value. This indicates the following picture about the development of vortex tangles. Initially, the tangle has vortex rings at a unique abundance ratio with respect to their sizes before the vortex line length is saturated. Consequently, the tangle develops while maintaining the abundance ratio of the vortex rings. Finally, both the ratio and the vortex line lengths of the tangle are saturated.

Figures 7(c) and 7(d) show the saturated vortex length distributions in some different parameters for parallel injection and spherical injection, respectively. Surprisingly, for each generating method, their behaviors are common, although the injection sizes, the injection frequencies, and the vortex line densities are substantially different.

The tangles by parallel injection show the power law in the range above the injection size. These slopes are $-1.67 \sim$ -1.98 . However, in the range below the injection size, any power law disappears. Too small vortex rings can be ignored because they are closer to the spatial resolution.

(b)

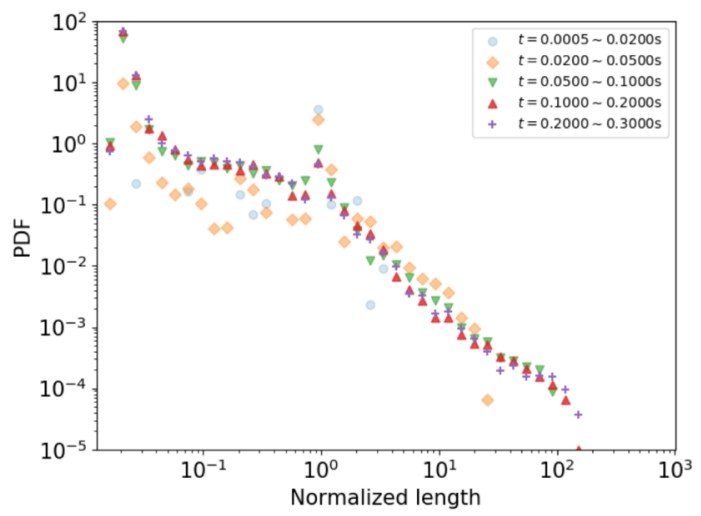

(d)

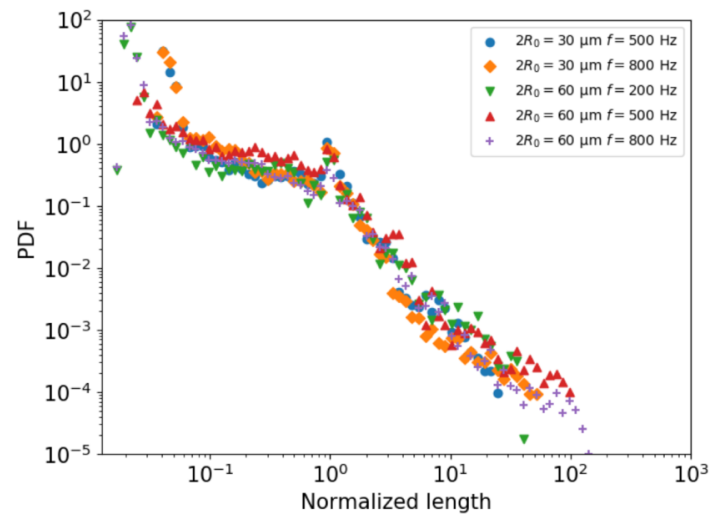

FIG. 7. The vortex length distribution. (a) and (c) "Parallel injection;" (b) and (d) "spherical injection;" (a) and (b) are the time development of the vortex number distribution averaged in the time interval in these legends. Panels (c) and (d) are the averaged vortex number distributions after $0.2 \mathrm{~s}$ under different conditions. 
The tangles by spherical injection do not clearly show any power law. The distributions in the range above the injection sizes are similar to an exponential distribution. These values in the range below the injection sizes also do not show the power law.

\section{CONCLUSION}

We numerically investigate the fractal dimension and the vortex length distribution of the localized tangles as a consequence of our previous study [28]. The fractal dimension increases with the vortex line density in the case of lowdensity tangles, whereas the dimension of the high-density tangle is saturated. The vortex length distribution of the tangles by parallel injection shows the power law though the distribution of the tangle by spherical injection does not.

The aim of this series of studies is to associate the internal structure of the localized vortex tangle with the self-similarity of the vortex emission [27,28]. We considered that the vortex length distribution enabled us to discuss it quantitatively. However, the self-similarity of the vortex emission is observed in the range of approximately 3 to $60 \mu \mathrm{m}$ in our previous study [28], and the self-similarity of the vortex length distribution is not observed in this range. The statistics of the internal structure do not necessarily correspond to that of the emitted vortex rings.

Although this simulation is performed at $T=0 \mathrm{~K}$, we will comment on the effects of finite temperatures. Kivotides et al. calculated the fractal dimension of vortex tangles at 1.3 and $1.9 \mathrm{~K}$ and found that it did not depend on the temperature [30]. When the mutual friction works at finite temperatures, the fine structure of a vortex tangle is smoothed out. A fine structure smaller than the intervortex distance does not affect the fractal dimension of the vortex tangles. Therefore, the fractal dimension should depend less on the temperature when it is given by a function of the vortex line density.

\section{ACKNOWLEDGMENTS}

M.T. was acknowledged by JSPS KAKENHI (Grant No. JP20H01855). S.I. was supported by a Grant-in-Aid for JSPS Research Fellows (Grant No. JP20J23131).
[1] U. Frisch, Turbulence: The Legacy of A. N. Kolmogorov (Cambridge University Press, Cambridge, UK, 1995).

[2] P. A. Davidson, Turbulence: An Introduction for Science and Engineers (Oxford University Press, Oxford, UK, 2004).

[3] W. F. Vinen and J. J. Niemela, J. Low Temp. Phys. 128, 167 (2002).

[4] M. Tsubota, M. Kobayashi, and H. Takeuchi, Phys. Rep. 522, 191 (2013).

[5] C. F. Barenghi, L. Skrbek, and K. R. Sreenivasan, Proc. Natl. Acad. Sci. USA 111, 4647 (2014).

[6] M. Tsubota, K. Fujimoto, and S. Yui, J. Low Temp. Phys. 188, 119 (2017).

[7] L. Tisza, Nature (London) 141, 913 (1938).

[8] L. Landau, Phys. Rev. 60, 356 (1941).

[9] W. F. Vinen, Proc. R. Soc. London A 260, 218 (1961).

[10] C. Nore, M. Abid, and M. E. Brachet, Phys. Rev. Lett. 78, 3896 (1997).

[11] C. Nore, M. Abid, and M. E. Brachet, Phys. Fluids 9, 2644 (1997).

[12] J. Maurer and P. Tabeling, Europhys. Lett. 43, 29 (1998).

[13] S. R. Stalp, L. Skrbek, and R. J. Donnelly, Phys. Rev. Lett. 82, 4831 (1999).

[14] M. Kobayashi and M. Tsubota, Phys. Rev. Lett. 94, 065302 (2005).

[15] M. Kobayashi and M. Tsubota, J. Phys. Soc. Jpn. 74, 3248 (2005).

[16] J. Jäger, B. Schuderer, and W. Schoepe, Phys. Rev. Lett. 74, 566 (1995).

[17] J. Luzuriaga, J. Low Temp. Phys. 108, 267 (1997).

[18] H. Yano, N. Hashimoto, A. Handa, M. Nakagawa, K. Obara, O. Ishikawa, and T. Hata, Phys. Rev. B 75, 012502 (2007).

[19] N. Hashimoto, R. Goto, H. Yano, K. Obara, O. Ishikawa, and T. Hata, Phys. Rev. B 76, 020504(R) (2007).
[20] D. Garg, V. B. Efimov, M. Giltrow, P. V. E. McClintock, L. Skrbek, and W. F. Vinen, Phys. Rev. B 85, 144518 (2012).

[21] D. I. Bradley, S. N. Fisher, A. M. Guénault, R. P. Haley, V. Tsepelin, G. R. Pickett, and K. L. Zaki, J. Low Temp. Phys. 154, 97 (2009).

[22] D. Bradley, M. Fear, S. Fisher, A. Guénault, R. Haley, C. Lawson, P. McClintock, G. Pickett, R. Schanen, V. Tsepelin, and L. Smethurst, J. Low Temp. Phys. 156, 116 (2009).

[23] H. Yano, Y. Nago, R. Goto, K. Obara, O. Ishikawa, and T. Hata, Phys. Rev. B 81, 220507(R) (2010).

[24] D. I. Bradley, A. M. Guénault, S. N. Fisher, R. P. Haley, M. J. Jackson, D. Nye, K. O’Shea, G. R. Pickett, and V. Tsepelin, J. Low Temp. Phys. 162, 375 (2011).

[25] D. I. Bradley, S. N. Fisher, A. M. Guénault, R. P. Haley, M. Kumar, C. R. Lawson, R. Schanen, P. V. E. McClintock, L. Munday, G. R. Pickett, M. Poole, V. Tsepelin, and P. Williams, Phys. Rev. B 85, 224533 (2012).

[26] D. I. Bradley, M. Človečko, S. N. Fisher, D. Garg, E. Guise, R. P. Haley, O. Kolosov, G. R. Pickett, V. Tsepelin, D Schmoranzer, and L. Skrbek, Phys. Rev. B 85, 014501 (2012).

[27] H. Yano, K. Sato, K. Hamazaki, R. Mushiake, K. Obara, and O. Ishikawa, J. Low Temp. Phys. 196, 184 (2019).

[28] T. Nakagawa, S. Inui, M. Tsubota, and H. Yano, Phys. Rev. B 101, 184515 (2020).

[29] J. C. Vassilicos and J. G. Brasseur, Phys. Rev. E 54, 467 (1996).

[30] D. Kivotides, C. F. Barenghi, and D. C. Samuels, Phys. Rev. Lett. 87, 155301 (2001).

[31] T. Araki, M. Tsubota, and S. K. Nemirovskii, Phys. Rev. Lett. 89, 145301 (2002).

[32] S. Fujiyama and M. Tsubota, J. Low Temp. Phys. 158, 428 (2009).

[33] K. W. Schwarz, Phys. Rev. B 31, 5782 (1985).

[34] J. C. H. Fung and J. C. Vassilicos, Phys. Rev. E 57, 1677 (1998), 